\title{
Experimental Membranous Glomerulonephritis in Rats
}

\author{
QUANTITATIVE STUDIES OF GLOMERULAR IMMUNE DEPOSIT \\ FORMATION IN ISOLATED GLOMERULI AND WHOLE ANIMALS
}

\author{
David J. Salant, Christine Darby, and William G. Couser, Evans Memorial \\ Department of Clinical Research and Department of Medicine, University \\ Hospital, Boston University Medical Center, Boston, Massachusetts 02118
}

\begin{abstract}
A B S T R A C T Quantitation of immune deposit formation in glomeruli and correlation with immunohistologic and functional changes has been accomplished only in models of anti-glomerular basement membrane antibody-induced nephritis, or indirectly in immune complex disease by measuring radiolabeled antigen deposition. The kinetics of subepithelial immune deposit formation and the relationship between the quantity of antibody deposited and proteinuria are defined here for the first time in an established model of membranous immune complex nephritis (passive Heymann nephritis) induced by a single intravenous injection of ${ }^{125}$ I-labeled sheep immunoglobulin (Ig)G antibody to rat tubular brush border antigen (FxlA). Measurement of antibody deposition in glomeruli $(\mathrm{GAb})$ isolated from rats injected with $10 \mathrm{mg}$ of antiFxlA demonstrated a mean of $12 \mu \mathrm{g} \mathrm{GAb}$ in $4 \mathrm{~h}$, which increased linearly to $48 \mu \mathrm{g}$ in $5 \mathrm{~d}$. GAb represented only 20 and $44 \%$ of total kidney antibody binding at these times. Proteinuria occurred only after 4-5 d of antibody deposition in rats with total kidney antibody binding exceeding $\sim 200 \mu \mathrm{g} / 2$ kidneys. Steroid treatment and vasoactive amine blockade did not significantly alter the quantity or localization of immune deposits. It was also demonstrated that isolated rat glomeruli specifically bound nephritogenic quantities of anti-FxlA in vitro within hours. Analysis of the quantitative aspects of glomerular antibody deposition in vivo and glomerular antibody binding in vitro pro-
\end{abstract}

Portions of this work were presented at the 11th Annual Meeting of the American Society of Nephrology, New Orleans, La., 21 November 1978, and have been published in abstract form in 1978. Kidney Int. 14: 717.

Dr. Salant is the recipient of a National Institutes of Health Clinical Investigator Award (AM 00742). Dr. Couser is the recipient of a National Institute of Health Research Career Development Award (AM 00102).

Received for publication 10 May 1979 and in revised form 14 March 1980. vides additional evidence that subepithelial immune deposits in passive Heymann nephritis may form in situ by reaction of free antibody with antigenic constitutents of the normal rat glomerulus. The observed kinetics of deposit formation differ markedly from those in anti-glomerular basement membrane disease and suggest a role for factors in addition to antigen-antibody interaction in determining this unique pattern of glomerular immune deposit formation.

\section{INTRODUCTION}

Membranous nephropathy is a well-characterized glomerular disease in which the gradual accumulation of deposits of immunoglobulin and complement in a discontinuous granular pattern in the subepithelial space results in a marked increase in capillary permeability with proteinuria and nephrotic syndrome $(1,2)$. Unlike most other types of glomerulonephritis mediated by immune deposits, in membranous nephropathy there are no significant histologic inflammatory changes early in the disease and renal function is generally not compromised when proteinuria begins. Although the interpretation of several studies has been that deposits of immunoglobulin in a membranous pattern result from glomerular trapping of low molecular weight immune complexes formed in the circulation (3-6), evidence obtained from recent studies in a rat model that resembles membranous nephropathy suggests that these deposits may form in situ as a result of free antibody interacting with antigens normally present or fixed in the glomerular capillary wall (7-11). This hypothesis would predict that the kinetics of glomerular antibody deposition and the functional consequences thereof should resemble those described in models of nephritis mediated by antibody to glomerular basement membrane antigens (nephrotoxic nephritis) in which glomerular antibody deposition is immediate (12) and induces an acute inflamma- 
tory lesion apparently similar to the experimental Arthus reaction accompanied by significant alterations in renal function $(13,14)$. Whether the marked differences in these two types of glomerular lesions reflect differences in the mechanisms of deposit formation or quantitative differences in the rate or amount of antibody deposition is unclear.

In passive Heymann nephritis ( $\mathrm{PHN}),{ }^{1}$ rats injected with heterologous antibody to an antigen derived from the brush border of proximal tubular epithelial cells (Fx1A) develop subepithelial deposits of immunoglobulin (Ig)G and host complement and a glomerular lesion indistinguishable by immunofluorescence (IF) and electron microscopy (EM) from membranous nephropathy in man $(8-11,15)$. The capacity to induce subepithelial deposits by a single injection of antibody has afforded the opportunity to measure quantitatively the kinetics of glomerular immune deposit formation and to correlate glomerular antibody deposition with proteinuria for the first time in an experimental model of membranous nephropathy. Although some quantitative data on glomerular subepithelial immune deposits has been obtained in the serum sickness models in rabbits, these studies have measured only antigen deposition (16). All information currently available relating antibody deposits in glomeruli to alterations in glomerular histology and function has been derived from studies in nephrotoxic nephritis models (12-14).

These studies provide the first quantitative information on the rate and quantity of antibody deposition in experimental membranous nephropathy as they relate to changes in capillary wall permeability and define a unique time-course of glomerular immune deposit formation, which may explain some of the unique features of this common glomerular disease. In vitro studies demonstrate that isolated rat glomeruli can bind sufficient antibody to account for the deposits seen in vivo in proteinuric rats. Moreover, in vivo measurements of antibody binding after steroid therapy and vasoactive amine blockade show no effect of these manipulations on deposit formation.

\section{METHODS}

\section{Preparation of antibody to rat FxIA and glomerular basement membrane (GBM) and induction of $\mathrm{PHN}$}

FxlA was prepared from fresh, saline-perfused, rat renal cortices as described by Edgington et al. (17). Adult male sheep were immunized three or four times with $75-100 \mathrm{mg}$

${ }^{1}$ Abbreviations used in this paper: EM, electron microscopy; Fx1A, proximal tubular epithelial cell brush border antigen; GAb, glomerular antibody binding; GBM, glomerular basement membrane; IF, immunofluorescence; $\mathrm{PHN}$, passive Heymann nephritis; TKAb, total kidney antibody binding.
FxlA in complete Freund's adjuvant (Difco Laboratories, Detroit, Mich.) over a 2 -mo period. Serum collected 2 wk after the last immunization was heat-inactivated $\left(56^{\circ} \mathrm{C}, 30\right.$ $\mathrm{min}$ ) and absorbed with pooled rat serum, leukocytes, erythrocytes, and platelets as described (7). To avoid contaminating the radiolabeled IgG with rat IgG, absorption with rat serum was performed after radiolabeling (see below). The IgG fraction was isolated from a $50 \%$ ammonium sulfate precipitate by ion-exchange chromatography on DEAE-Sephadex (Pharmacia Fine Chemicals, Inc., Piscataway, N. J.). AntiFxlA IgG, at a concentration of $10 \mathrm{mg} / \mathrm{ml}$, was not reactive with rat plasma by micro-Ouchterlony (18) and immunoelectrophoresis (19), but made two precipitin lines against a $10 \mathrm{mg} / \mathrm{ml}$ suspension of $\mathrm{Fx} 1 \mathrm{~A}$ in saline $(17,20)$. By indirect IF, the antibody reacted with rat proximal tubular epithelial cell brush border to a dilution of $1 \times 10^{6}$.

Antibody to rat GBM was prepared by immunizing sheep with whole rat glomeruli as described (21). IgG isolation, absorption, and radiolabeling were carried out exactly as described for anti-Fx1A. A dose of $10 \mathrm{mg}$ of anti-GBM IgG administered to normal rats induced $4+$ linear staining for IgG on the GBM, no extraglomerular deposits by IF, and urine protein excretion of about $200 \mathrm{mg} / \mathrm{d}$ in the $24-\mathrm{h}$ period after injection. Normal sheep IgG was isolated from normal sheep serum and radiolabeled as described below. Intravenous administration to normal rats produced no renal deposits by IF up to $\mathbf{5} \mathrm{d}$ later.

PHN was induced in male Charles River rats weighing 190-220 g (Charles River Breeding Laboratories, Wilmington, Mass.) by intravenous injection of anti-FxlA. All studies were carried out for periods of up to $5 \mathrm{~d}$ after antibody administration.

\section{Radiolabeling of anti-FxIA IgG, anti-GBM IgG, and normal sheep IgG with ${ }^{125}$ I}

Sheep IgG, isolated as described above from normal sheep serum or antiserum to rat FxlA or GBM, was labeled with ${ }^{125}$ I using the chloramine $\mathrm{T}$ method as modified by McConahey and Dixon (22). Aliquots of $5 \mathrm{mg} \mathrm{IgG}$ were labeled. $10 \mathrm{mg}$ of unlabeled IgG was added before removal of free ${ }^{125} \mathrm{I}$ by ion-exchange chromatography on AG1-X8 (Bio-Rad Laboratories, Richmond, Calif.) and either extensive dialysis or Sephadex G-25 chromatography. Precipitation of the final preparation with $10 \%$ TCA showed that 89-93\% of the isotope was protein bound, and the specific activity was $1-5 \times 10^{6}$ cpm/mg IgG (after correction for TCA precipitability). The labeled preparations were stored at $-70^{\circ} \mathrm{C}$ until use, when they were further diluted with the appropriate unlabeled IgG (normal, anti-Fx1A, or anti-GBM) to the desired amount of protein and $\sim 1 \times 10^{6} \mathrm{cpm} / 10 \mathrm{mg}$ IgG. Measurement of the final specific activity was determined immediately before use. Labeled anti-FxlA reacted similarly to the unlabeled antibody described above by precipitin analysis and indirect IF. Pilot studies demonstrated no apparent difference by IF or EM between glomerular deposits induced in vivo with equal amounts of ${ }^{125}$ I-labeled and unlabeled anti-Fx1A.

\section{In vitro glomerular binding of antibody}

Experiment A. The ability of anti-FxlA to bind to fresh isolated glomeruli in vitro was compared to that of normal sheep IgG (negative control) and anti-GBM (positive control). Glomeruli were isolated immediately from kidneys perfused in vivo with heparinized saline (see below) by differential sieving through Nos. 150 and 200 mesh brass sieves (Sargent-Welch Co., Skokie, Ill.), washed extensively 
with cold, $0.02 \mathrm{M}$ phosphate-buffered saline, $\mathrm{pH} 7.2$, and suspended in Eagle's minimal essential medium supplemented with L-glutamine, penicillin, and streptomycin (Gibco Laboratories, Grand Island Biological Co., Grand Island, N. Y.). Glomeruli prepared in this way had $<1 \%$ identifiable tubules by light microscopy, and $43 \%$ were encapsulated (determined by counting $1 \times 10^{3}$ glomeruli). Six $10 \times 75-\mathrm{mm}$ glass test tubes containing $0.5-\mathrm{ml}$ aliquots of a uniform glomerular suspension were incubated with $0.5 \mathrm{ml}$ of the appropriate labeled sheep IgG at a final concentration of $0.5 \mathrm{mg} / \mathrm{ml}$ at $37^{\circ} \mathrm{C}$ with constant shaking. Two tubes were removed, centrifuged, the glomeruli washed twice in phosphate-buffered saline, and counted after $1 \mathrm{~h}$ of incubation. Glomeruli in the remaining tubes were washed and counted after $21 \mathrm{~h}$ incubation. Radioactivity was determined in a well-type Packard auto-gamma spectrometer, model 3002 (Packard Instrument Co. Inc., Downers Grove, Ill.). Glomerular bound IgG per tube was calculated by dividing counts per minute above background by the specific activity of the radiolabeled IgG preparation. The number of glomeruli per tube was determined at the conclusion of the experiment by fixing glomeruli in $10 \%$ formalin for $30 \mathrm{~min}$, suspension in $30 \%$ aqueous polyvinylpyrolidone (average mol wt 40,000, Sigma Chemical Co., St. Louis, Mo.), and visual counting in a FuchsRosenthal hemocytometer. Results for each experiment are expressed as micrograms of IgG bound per tube because all tubes contained equal numbers of glomeruli. To evaluate possible effects of restricted antibody penetration of intact glomeruli on the results observed, a second aliquot of the same glomerular suspension was disrupted by sonication on a Branson sonifier, model W-350 (Branson Sonic Power Co., Danbury, Conn.) at an output setting of 6 for 60 and $12 \mathrm{~s}$. Total disruption of glomeruli was confirmed by light microscopy. Four tubes containing the same number of sonicated glomeruli as those containing intact glomeruli were incubated in parallel with the three preparations of IgG as described above.

Experiment B. Within Bowman's capsule of some rat glomeruli are proximal tubular epithelial cells with brush borders that are reactive with anti-FxlA. To evaluate any possible effect of these cells on in vitro antibody binding, the experiment described above was repeated using a second preparation of fresh glomeruli, which still contained $<1 \%$ nonglomerular elements, but only $7 \%$ of the glomeruli were encapsulated. These glomeruli were prepared from the kidneys of Charles River rats weighing $350-400 \mathrm{~g}$ using Nos. 170 and 200 sieves and the addition of two low speed centrifugations ( $120 \mathrm{~g}$ for $90 \mathrm{~s}$ ) of the glomerular preparation during washing. In this second study, five tubes containing equal numbers of intact glomeruli were incubated with labeled anti-FxlA and with normal sheep IgG.

To assess the distribution of bound IgG, aliquots of isolated glomeruli that had previously been incubated for $21 \mathrm{~h}$ with sheep anti-Fx1A, anti-GBM, normal IgG, or medium alone, were suspended in fluorescein-conjugated rabbit antisheep IgG for $30 \mathrm{~min}$, washed three times in phosphatebuffered saline, and examined by IF. In parallel studies, anti-FxlA was incubated with fresh cryostat sections of normal rat kidney for $45 \mathrm{~min}$ before staining for sheep IgG.

\section{Disappearance kinetics of anti-FxlA and normal sheep IgG from rat blood and whole rats}

PNH was induced in 16 rats by injection of $10 \mathrm{mg}$ i.v. of labeled anti-Fx1A and the disappearance kinetics was determined by measuring the concentration of anti-FxlA IgG in rat blood at 1,4 , and $24 \mathrm{~h}$, and daily for $5 \mathrm{~d}$. A second group of
19 rats was given $10 \mathrm{mg}$ i.v. of labeled normal sheep IgG and the disappearance kinetics measured at the same time intervals. To assess the effect of anti-FxlA on the disappearance kinetics of normal sheep IgG, a third group of nine rats was given $10 \mathrm{mg}$ i.v. of unlabeled anti-FxlA IgG with a trace dose of labeled normal sheep IgG and the disappearance kinetics was measured at 1,24 , and $72 \mathrm{~h}$. The concentration of sheep IgG in rat blood (microgram per milliliter) was calculated from the radioactivity of $100 \mu \mathrm{l}$ of rat blood (corrected for background and decay) drawn from the tail and expressed as a percentage of the initial concentration. The initial concentration was calculated from the administered dose of IgG divided by the rat blood volume (estimated at $7 \%$ of body weight) (23) rather than by direct measurement because of difficulty obtaining blood immediately from antibody injected rats. All rats were given $0.005 \mathrm{~g} / 100 \mathrm{ml} \mathrm{KI}$ and $0.45 \mathrm{~g} / 100 \mathrm{ml} \mathrm{NaCl}$ in their drinking water to block thyroid uptake and facilitate the urinary excretion of free ${ }^{125} \mathrm{I}$.

Alterations in plasma volume were calculated from measurements of the hematocrit on blood samples taken before antibody injection and 1 and $4 \mathrm{~h}$ afterward. Persistence of free circulating anti-FxlA in blood taken at each time interval was determined by indirect IF on normal rat kidney.

Whole-body radioactivity was measured in a whole-animal Packard gamma spectrometer, model 3002 immediately after injection of radiolabeled anti-Fx1A or normal sheep IgG and daily for $5 \mathrm{~d}$. The amount of sheep IgG retained by each of four rats in both groups was determined from the whole body radioactivity at each time interval and expressed as a percentage of the activity measured immediately after injection.

\section{Kinetics of total kidney antibody binding (TKAb) in PHN}

PHN was induced in 16 rats by the injection of $10 \mathrm{mg}$ i.v. of labeled anti-FxlA and groups of three to four were killed at 1 and $4 \mathrm{~h}$, and 1,2 , and $5 \mathrm{~d}$ later for determination of the TKAb. Under ether anesthesia, the abdomen was opened, $100 \mathrm{U}$ i.v. heparin injected, the aorta ligated immediately above and below the renal arteries, and the kidneys gently perfused via the superior mesenteric artery and aorta with 60 $\mathrm{ml}$ heparinized saline over 3-5 min. The kidneys were then removed, stripped of their capsules and pedicles, and TKAb determined from kidney radioactivity in each rat. In a pilot study to evaluate this procedure, washed homogenates of six kidneys from rats injected with labeled anti-FxlA were found to contain $87.3 \pm 4.3 \%$ of the kidney bound radioactivity, whereas the activity in the washes was $91.5 \pm 5.2 \%$ TCA precipitable. To determine the amount of sheep IgG present due to blood not removed by perfusion or nonspecifically bound in the kidneys, sheep IgG was measured at the same time intervals in the kidneys of 19 rats injected with $10 \mathrm{mg}$ of labeled normal sheep IgG and at 1,24 , and $72 \mathrm{~h}$ in nine rats injected with $10 \mathrm{mg}$ of unlabeled anti-FxlA IgG with a trace amount of labeled normal sheep IgG. An equation derived from the demonstrated relationship between blood levels of normal sheep IgG and nonspecific sheep IgG content of perfused kidneys was then used to correct for nonimmunologically bound IgG in all measurements of TKAb (Results).

\section{Measurement of glomerular bound antibody (GAb)}

To determine the amount of TKAb specifically bound in glomeruli, glomeruli were isolated individually from the perfused kidneys of each rat by differential sieving as described 
for experiment $\mathrm{A}$ above, counted, and their radioactivity measured. The GAb of each rat was calculated from the equation: $\mathrm{GAb}$ (micrograms per two kidneys) = counts per minute in glomeruli $\times 76,000 /$ number of glomeruli $\times$ specific activity, where $76,000=$ number of glomeruli in two rat kidneys $(24,25)$. When glomeruli were isolated from the perfused kidneys of rats given normal sheep IgG, nonspecific contamination of glomeruli with sheep IgG was found to be $<1 \mu \mathrm{g} / 2$ kidneys at all time intervals and has therefore been disregarded in the determination of GAb.

\section{Relationship of antibody deposition to the development of proteinuria}

PHN was induced in 38 rats by intravenous injection of labeled anti-FxlA IgG in doses varying from 5 to $35 \mathrm{mg}$. Urine protein excretion was measured on 24-h urine specimens collected from all rats before killing at day 5 and TKAb was measured in each rat.

\section{Effect of corticosteroid administration and vasoactive amine antagonists on the quantity of renal antibody deposited}

Rats were randomly allocated to four groups of six animals per group. All were injected with $12.0 \mathrm{mg}$ of labeled antiFxlA and blood and kidneys were taken $5 \mathrm{~d}$ later for measurement of TKAb.

Group 1. Group 1 was an untreated control group.

Group 2. Group 2 received methylprednisolone, $10 \mathrm{mg} /$ $\mathrm{kg}$ i.p., at 12 -h intervals for $24 \mathrm{~h}$ before induction of PHN, and for $5 \mathrm{~d}$ thereafter.

Group 3. Group 3 received chlorpheniramine maleate, $15 \mathrm{mg} / \mathrm{kg}$ i.p., at 12 -h intervals starting $24 \mathrm{~h}$ before anti-FxlA injection, and then for $5 \mathrm{~d}$.

Group 4. Group 4 received cyproheptadine, $15 \mathrm{mg} / \mathrm{kg}$ i.p., on the same schedule as groups 2 and 3. The doses of chlorpheniramine maleate and cyproheptadine used have been shown to block local reaction to $1 \mu \mathrm{g}$ of histamine base or $1 \mu \mathrm{g}$ of serotonin (5-hydroxytryptamine) (11).

\section{Tissue processing, IF, and EM procedures}

Renal tissue obtained at death was divided into two portions. Tissue for IF was snap-frozen in dry ice-isopentane, and cryostat sections fixed in ether-ethanol were stained using techniques and controls described elsewhere $(7,26)$. IF staining used the fluorescein isothiocyanate conjugated IgG fractions of monospecific rabbit anti-sheep IgG and goat anti-rat IgG (N. L. Cappel Laboratories Inc., Cochranville, Pa.). Antibody to rat IgG was not cross-reactive with sheep IgG by immunoprecipitin or IF analysis. IF was evaluated and photographed on a Leitz Ortholux II microscope equipped with a Ploempak 2.2 vertical fluorescence illuminator (E. Leitz, Inc. Rockleigh, N. J.). EM studies were kindly performed by Dr. Magda Stilmant (Mallory Institute of Pathology, Boston City Hospital, Boston, Mass.) on two representative rats injected with labeled anti-FxlA to insure that radiolabeling did not alter the antibody localization. Tissue for EM was immersion-fixed in glutaraldehyde and post-fixed in osmium followed by en bloc staining in uranyl acetate for $30 \mathrm{~min}$ before embedding in Epon 812 (Shell Chemical Co., Houston, Tex.). Thin sections were studied and photographed on a Philips 300 electron microscope (Philips Electronic Instruments, Inc., Mahwah, N. J.).

\section{Other procedures}

Urine protein excretion was measured by a sulfosalicylic acid method on 24-h collections obtained in metabolic cages (27). Concentrations of purified sheep IgG were measured by the biuret method. Microhematocrit was measured on blood obtained from the tail vein using $100 \mu \mathrm{l}$ heparinized capillary tubes and a micro-capillary reader (Damon/IEC Div., Needham, Mass.).

\section{Statistical analysis}

Regression analysis and determination of the correlation coefficient were performed by standard methods $(28)$ on data relating nonspecific contamination of the kidneys with normal sheep IgG to blood concentration of normal sheep IgG and on the kinetics of the disappearance of sheep IgG from rat blood after semi-logarithmic transformation. Significance of the difference between the half-lives of normal sheep IgG and anti-FxlA IgG in rat blood was evaluated by comparison of the slopes (28). Students $t$ test for unpaired data was used to analyze the results of in vitro glomerular binding of antibody, and the differences between the levels of normal sheep IgG and anti-FxlA IgG remaining in rat blood and in whole animals (28). The effects of steroids and vasoactive amine blockers were examined by analysis of variance (28). Differences were regarded as significant when $P<0.05$. Unless otherwise stated, all values are expressed as the mean \pm 1 SD.

\section{RESULTS}

Localization of kidney deposits in PHN induced with labeled anti-FxlA. The glomerular lesion on day 1-5 was identical in rats injected with labeled anti-FxlA and nonradiolabeled antibody and was identical to findings in this model described in detail elsewhere $(10,11)$. Finely granular deposits of sheep IgG and rat C3 were present on the capillary walls of all glomeruli by IF, and electron-dense deposits were localized exclusively in the subepithelial space by EM. Trace deposits of rat IgG were noted by IF in glomeruli $5 \mathrm{~d}$ after anti-FxlA IgG administration. Renal deposition of sheep IgG was confined to glomeruli by IF for $48 \mathrm{~h}$ after antibody injection, after which some granular deposits were noted along tubular basement membranes as well. In addition, rats with heavy proteinuria at $5 \mathrm{~d}$ demonstrated binding of sheep antibody to proximal tubular brush borders. No renal deposition of normal sheep IgG was observed by IF in control rats.

Binding of normal sheep IgG, anti-FxlA, and antiGBM to isolated rat glomeruli in vitro. Incubation of isolated rat glomeruli with labeled normal sheep IgG, anti-FxlA, and anti-GBM demonstrated specific binding of both anti-FxlA IgG and anti-GBM IgG which was apparent within $1 \mathrm{~h}$ and had increased after $21 \mathrm{~h}$ (Table 1 , experiment $\mathrm{A}$ ). The amount of antiFxlA IgG bound was approximately six times greater than normal IgG $(P<0.001)$ and was not affected by sonic disruption of glomeruli $(P>0.05)$. Whole 
TABLE I

Binding of Normal Sheep IgG, Anti-FxlA and Anti-GBM

to Isolated Rat Glomeruli In Vitro

\begin{tabular}{lccc}
\hline & Normal IgG & Anti-FxIA IgG & Anti-GBM IgG \\
\hline & & $\mu g$ IgG/tube & \\
Experiment A & & & \\
$\quad$ Whole glomeruli & & & \\
$\quad 1$ h incubation & 4.1 and $4.1 \S$ & 16.2 and 21.6 & 34.0 and 26.9 \\
$\quad 21$ h incubation & $7.7 \pm 0.49(4)^{\prime \prime}$ & $46.1 \pm 1.55(4)$ & $81.6 \pm 1.75(4)$ \\
$\quad \begin{array}{l}\text { Sonicated glomeruli } \\
\quad 21 \text { h incubation }\end{array}$ & $6.4 \pm 0.01(4)$ & $47.4 \pm 0.92(4)$ & $90.8 \pm 0.04(4)$ \\
$\begin{array}{c}\text { Experiment B } \\
\text { Whole glomeruli }\end{array}$ & & & \\
$\quad 21$ h incubation & $5.6 \pm 0.13(5)$ & $34.0 \pm 0.57(5)$ & ND \\
\hline
\end{tabular}

\footnotetext{
${ }^{*}$ In experiment A each tube contained $20,900 \pm 1,430$ (5) glomeruli (whole or sonicated) of which $43 \%$ were encapsulated.

$\ddagger$ In experiment $B$ each tube contained 6,100 \pm 810 (6) whole glomeruli of which $7 \%$ were encapsulated.

$\S$ The values in two tubes.

"Mean \pm 1 SD (number of tubes).

I Not done.
}

glomeruli bound almost twice as much anti-GBM IgG as anti-FxlA $\operatorname{IgG}(P<0.001)$, and sonication produced an increase of $11 \%$ in the binding of anti-GBM IgG $(P<0.001)$.

The amount of anti-Fx1A IgG bound in experiment $B$ (Table I) was also found to be six times greater than normal IgG $(P<0.001)$ despite reduction of the percentage of encapsulated glomeruli from $43 \%$ in experiment $\mathrm{A}$ to $7 \%$ in experiment $\mathrm{B}$. The decrease in absolute binding of anti-FxlA IgG in experiment B is largely related to the smaller number of glomeruli per tube. Examination of whole glomeruli from each group by indirect IF for sheep IgG showed diffuse binding of anti-Fx IA IgG to the capillary walls of decapsulated glomeruli (Fig. 1). The exact site of binding could not be identified by IF. Anti-GBM IgG was seen in a linear pattern on the GBM, whereas normal sheep IgG showed very weak diffuse staining of glomeruli (Fig. 1). Glomeruli incubated in buffer alone and fresh glomeruli failed to show specific fluorescence after staining with conjugated anti-sheep IgG. Indirect IF of anti-FxlA IgG on cryostat sections of normal rat kidney produced intense staining of proximal tubular brush borders and faint, diffuse glomerular capillary wall staining without distinct granularity.

Disappearance kinetics of normal sheep IgG and anti-FxlA from rat blood and whole rats. After injection of $10 \mathrm{mg}$ of labeled anti-Fx1A or normal sheep IgG, an exponential decline in the concentration of sheep IgG in rat blood was observed (Fig. 2). The initial loss of anti-FxlA IgG from the circulation was significantly greater than that of normal IgG. At $1 \mathrm{~h}$
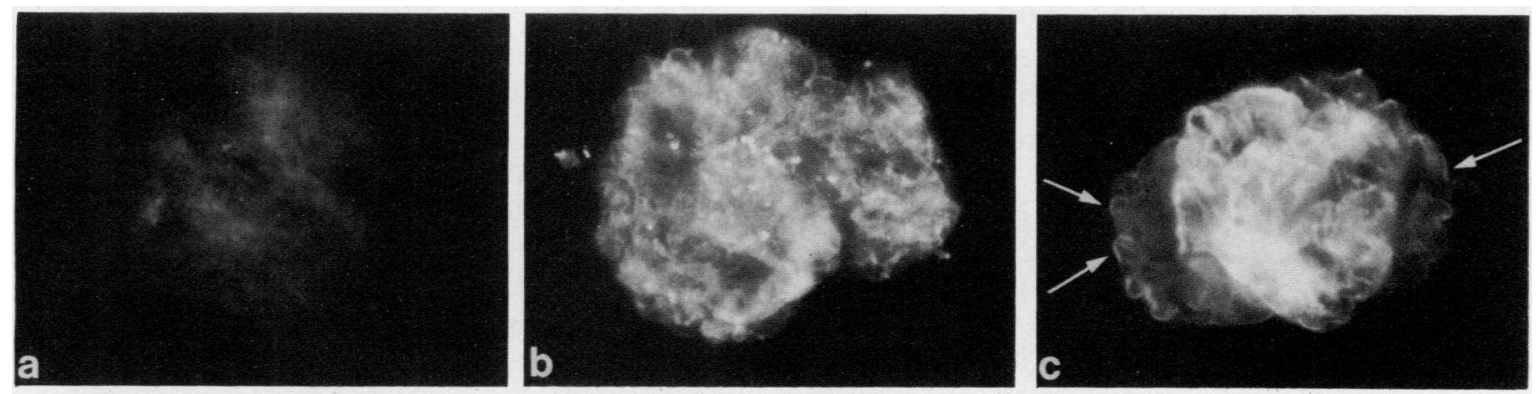

FIGURE 1 Isolated rat glomeruli after $21 \mathrm{~h}$ of incubation with equal amounts of normal sheep IgG (a), sheep anti-FxlA IgG (b), and sheep anti-GBM IgG (c), stained with fluorescein conjugated rabbit anti-sheep IgG. Diffuse binding of anti-FxlA IgG and anti-GBM IgG is apparent as compared to very weak staining of normal IgG. In some areas anti-GBM IgG appears to bind to the capillary wall in a linear pattern (arrows). All exposures are $10 \mathrm{~s} .(\times 250$.) 


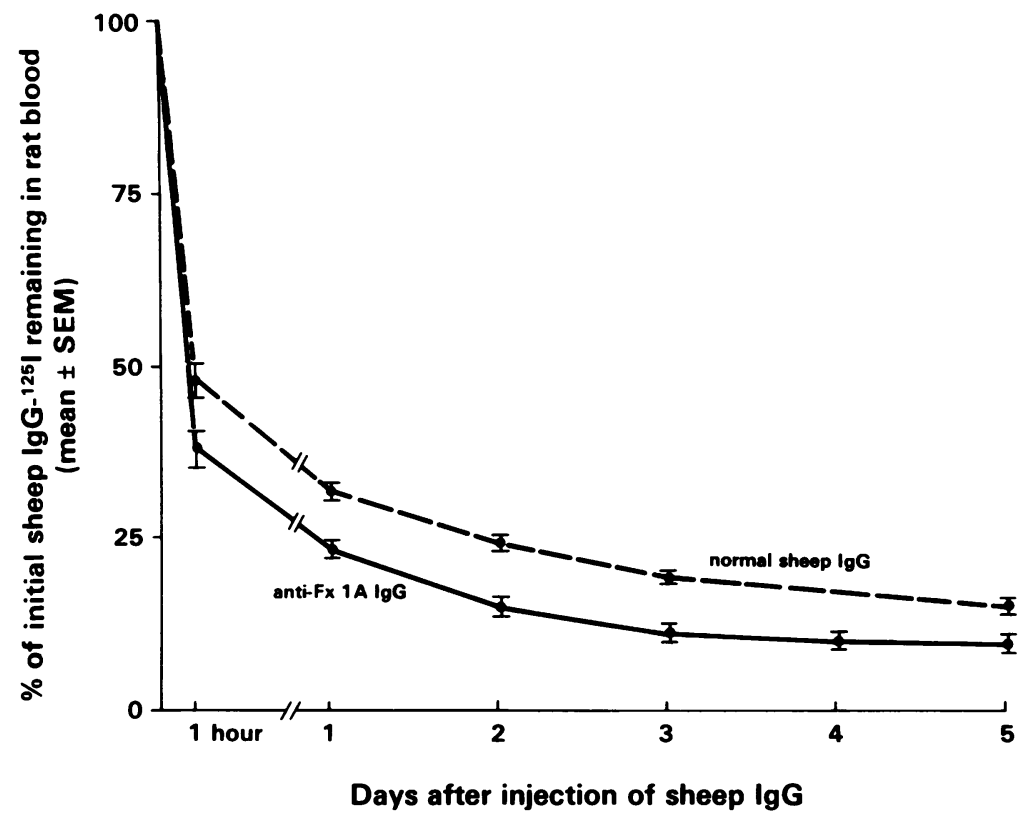

FIGURE 2 Disappearance kinetics of sheep IgG from the blood of rats injected with $10 \mathrm{mg}$ of labeled normal sheep IgG or anti-FxlA IgG.

after injection, the concentration of sheep IgG in rat blood represented $37.9 \pm 6.5 \%(n=6)$ of the initial concentration in rats given anti-Fx1A as compared to 47.8 $\pm 5.2 \%(n=4)$ in the group given normal sheep IgG $(P<0.05)$ (Fig. 2). This discrepancy occurred during a period of markedly increased vascular permeability in antibody-injected animals. During the first hour after antibody injection, a $36 \%$ rise in hematocrit, from $42.2 \pm 1.4 \%$ to $57.3 \pm 1.9 \%$, was noted. Hematocrits had almost returned to pre-antibody injection levels by $4 \mathrm{~h}$ $(46.2 \pm 3.3 \%)$. Similar changes in hematocrit were not seen in the group given normal sheep IgG. From $1 \mathrm{~h}$ to $5 \mathrm{~d}$, disappearance kinetics were similar in both groups, with anti-FxlA $\operatorname{IgG}\left(t^{1} 1 / 2=2.2 \mathrm{~d}\right)^{2}$ disappearing slightly more rapidly than normal sheep $\operatorname{IgG}\left(t^{1} / 2=2.8 \mathrm{~d}\right)^{2}$ (Fig. 2). Rats given simultaneous unlabeled anti-FxlA and labeled normal sheep IgG had a $32 \%$ rise in hematocrit from $45.2 \pm 1.2 \%$ to $59.5 \pm 4.0 \%$, whereas the disappearance kinetics of normal sheep IgG were similar to those of rats given normal sheep IgG alone: percentage of initial sheep IgG remaining in rat blood, anti-Fx1A IgG plus labeled normal sheep IgG vs. labeled normal sheep IgG alone, at $1 \mathrm{~h} 45.2 \pm 5.2 \mathrm{vs}$. $47.8 \pm 5.2 \%$, at $24 \mathrm{~h} 28.2 \pm 5.1$ vs. $31.2 \pm 2.4 \%$, at $72 \mathrm{~h}$ $13.2 \pm 4.1$ vs. $18.3 \pm 1.0 \%$. In all animals injected with anti-Fx1A, blood taken at all time intervals up to and including day 5 contained free antibody as shown by $4+$

\footnotetext{
${ }^{2}$ The half-lives were determined from the slopes of the regression equations after semi-logarithmic transformation of the data shown in Fig. 2 (normal sheep IgG $r=-0.95$, antiFxlA IgG $r=-0.91$. Difference between the slopes $P<0.05$ ).
}

staining of proximal tubular epithelial cell brush border of normal rat kidney by indirect IF.

The decline of whole animal activity was significantly greater at all time intervals in rats given antiFxlA than in those given normal sheep IgG. The percentage of the initial activity remaining after $24 \mathrm{~h}$ was $52.8 \pm 4.6 \%(n=4)$ for those given anti-FxlA and $75.5 \pm 3.9 \%(n=4)$ for those given normal sheep IgG, whereas at $5 \mathrm{~d} \quad 11.9 \pm 2.9 \%(n=4)$ and $38.9 \pm 4.8 \%$ $(n=4)$, respectively remained $(P<0.001)$.

$T K A b$ and GA $b$ of normal sheep IgG and anti-FxIA. Nonspecific contamination of the kidneys with normal sheep IgG in 19 rats injected with labeled normal sheep IgG was shown to be directly proportional to the blood concentration of sheep IgG $(\mathrm{r}=0.96, \log y=0.1642$ $+0.0034 \mathrm{x}, P<0.001$, where $\mathrm{x}=$ sheep IgG micrograms per milliliter rat blood and $\mathrm{y}=$ sheep IgG micrograms per two kidneys) (Fig. 3). Kidneys perfused and removed $1 \mathrm{~h}$ after injection of $10 \mathrm{mg}$ of labeled normal sheep IgG were contaminated with 29.9 $\pm 2.7 \mu \mathrm{g}$ sheep IgG/2 kidneys, and at $5 \mathrm{~d}$ this had fallen to $3.8 \pm 0.8 \mu \mathrm{g} / 2$ kidneys. The amount of nonspecific contamination with normal IgG was not increased by the concurrent administration of anti-FxlA. Therefore, in all studies using labeled anti-FxlA IgG, the calculation of TKAb incorporated a correction for nonspecific contamination using the regression equation shown above. Studies of washed glomeruli isolated from these kidneys showed nonspecific contamination with sheep IgG to be $<1 \mu \mathrm{g} / 2$ kidneys at all time intervals, and has therefore been disregarded in the determination of GAb. 


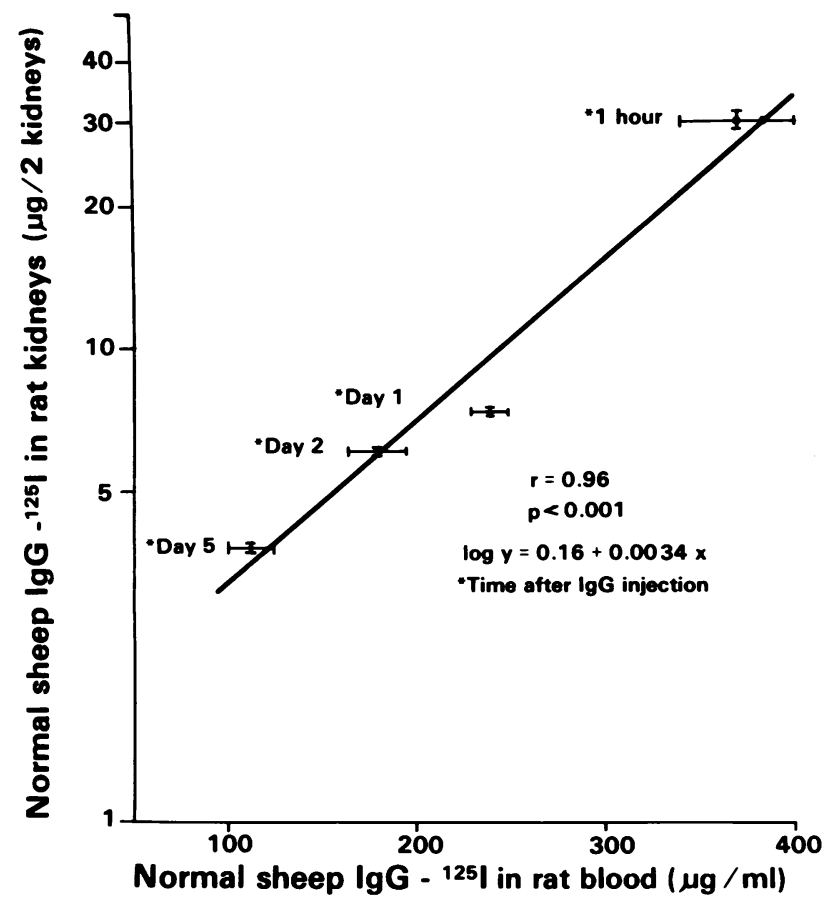

Figure 3 Relationship between sheep IgG content of perfused rat kidneys and simultaneous blood concentration of sheep IgG in 19 rats injected with $10 \mathrm{mg}$ normal sheep IgG and sacrificed at $1 \mathrm{~h}$, and 1,2 , and $5 \mathrm{~d}$. Mean $\pm \mathrm{SEM}$.

Fig. 4 depicts the kinetics of TKAb and GAb over $5 \mathrm{~d}$ in a group of 16 rats injected with $10 \mathrm{mg}$ of radiolabeled anti-Fx 1 A IgG. Initial kidney fixation was rapid with a mean of $61 \mu \mathrm{g}$ sheep IgG/2 kidneys bound within $4 \mathrm{~h}$. Thereafter antibody binding continued, albeit more slowly, over the 5-d study period. The mean TKAb at $5 \mathrm{~d}$ was $108 \mu \mathrm{g}$ sheep IgG/2 kidneys which represented $1.1 \%$ of the administered dose of antiFxlA IgG. Isolation of glomeruli and quantitation of GAb from the same kidneys showed that between $20 \%$ (at $4 \mathrm{~h}$ ) and $44 \%$ (at $5 \mathrm{~d}$ ) of TKAb was glomerular bound (Fig. 4). The kinetics of GAb were similar to those of TKAb although GAb assumed a greater proportion of TKAb with time. None of these rats became proteinuric within $5 \mathrm{~d}$.

Relationship of antibody deposition to the development of proteinuria. In 38 rats injected with doses of anti-FxlA IgG ranging from 5 to $35 \mathrm{mg}, 24-\mathrm{h}$ urine protein excretion was measured before determination of TKAb on day 5 . The upper $99 \%$ confidence limit of normal urine protein excretion determined in 20 normal, age-matched rats of similar weight was $3.5 \mathrm{mg} /$ 24 h. Fig. 5 shows the relationship between urine protein excretion and the amount of antibody deposited. Most rats (23/27) with TKAb of $200 \mu \mathrm{g} / 2$ kidneys or more had increased urine protein excretion (Fig. 5). Of 11 rats with TKAb of $<200 \mu \mathrm{g} / 2$ kidneys, only $3 \mathrm{had}$ mildly elevated urine protein excretion (Fig. 5 ).

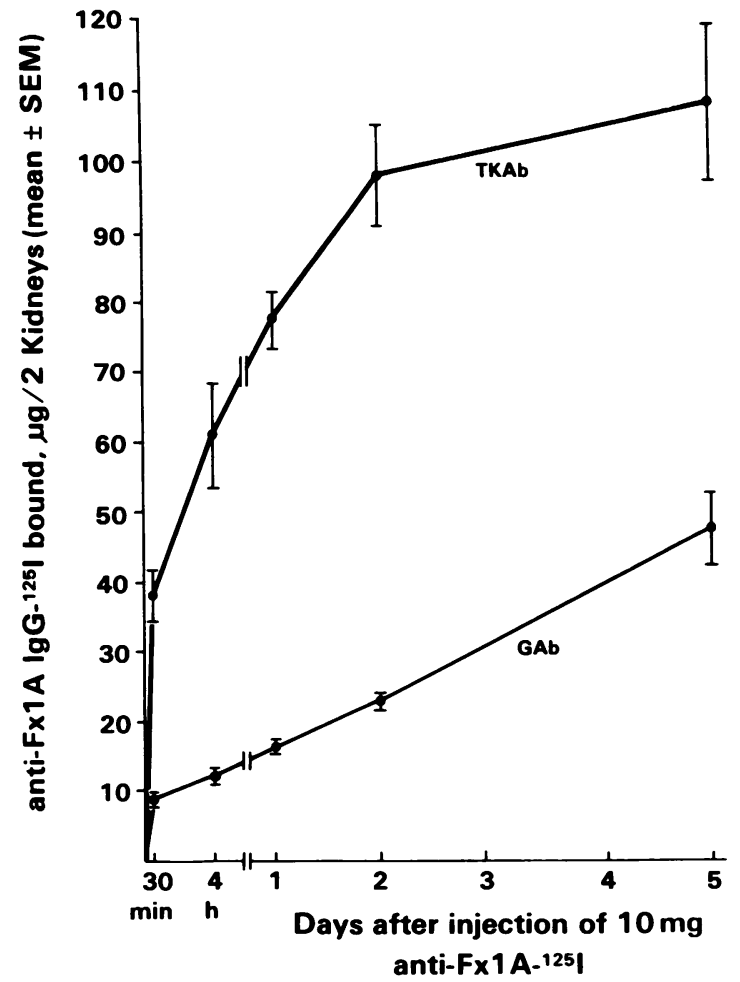

FIgURE 4 TKAb and GAb of antibody in 16 rats injected with $10 \mathrm{mg}$ of labeled anti-Fx1A IgG and killed after $30 \mathrm{~min}$, $4 \mathrm{~h}$, and 1,2 , and $5 \mathrm{~d}$. GAb was measured in glomeruli isolated from the same kidneys in which TKAb was measured.

Effect of corticosteroid administration and vasoactive amine antagonists on the quantity of renal antibody deposited. When rats were given methylprednisolone, chlorpheniramine maleate, or cyproheptadine starting $24 \mathrm{~h}$ before induction of PHN with $12.0 \mathrm{mg}$ of labeled anti-FxlA and continuing until killing $5 \mathrm{~d}$

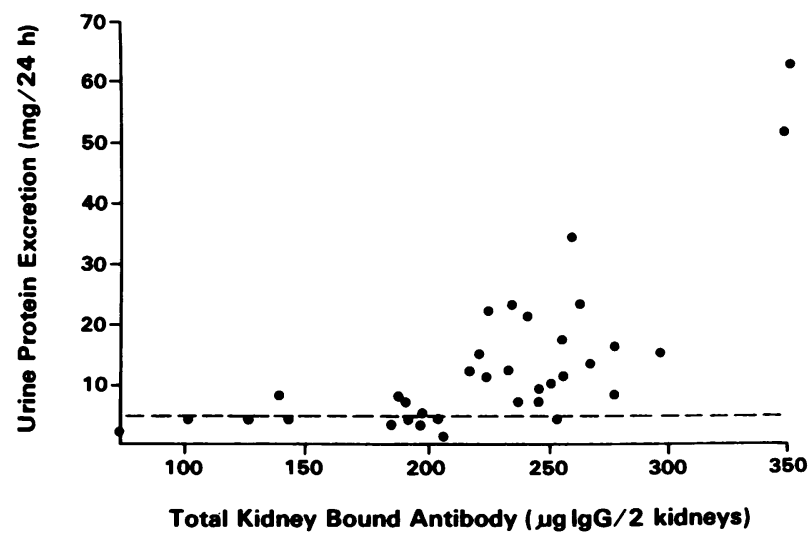

FIGURE 5 Relationship between 24-h urine protein excretion and total kidney antibody binding $5 \mathrm{~d}$ after administration of 5-35 mg of labeled anti-FxlA IgG to 38 rats. The dashed line represents the upper $99 \%$ confidence limit of 24 -h urine protein excretion in 20 normal age- and weight-matched rats. 
later, no effect on TKAb as compared to controls was observed on day 5. (Controls, 101.6 \pm 17 .3; methylprednisolone, 96.8 \pm 10.5 ; chlorpheniramine maleate, 102.7 \pm 10.7 ; cyproheptadine, 87.5 $\pm 5.4 \mu \mathrm{g}$ IgG/2 kidneys; $n=6$ for each group. Analysis of variance $P>0.05)$. In addition, no effect of methylprednisolone or vasoactive amine blockers on the localization of immune deposits was noted by IF.

\section{DISCUSSION}

Our previous studies in this model, and in autologous immune complex nephropathy (Heymann nephritis), have shown that intrinsic properties of the glomerulus are critical determinants of subepithelial deposit formation, and that these deposits may form in situ in the absence of circulating immune complexes $(7,10,11)$ as first suggested by Van Damme et al. (9). The present study is the first quantitative analysis of glomerular immune deposition in an established model of membranous nephropathy and demonstrates that nephritogenic amounts of an antibody, which induces granular subepithelial deposits in vivo, will also bind directly to isolated rat glomeruli in vitro. Despite apparent similarities demonstrated between PHN and antiGBM antibody nephritis with respect to the mechanism of glomerular antibody localization and the quantity of deposited antibody required to induce proteinuria, the kinetics of antibody deposition in PHN measured in this study differ markedly from those previously found in nephrotoxic nephritis (12) and suggest that factors in addition to antigen-antibody interaction are involved in determining this unique pattern of immune deposit formation.

Two technical aspects of the study deserve comment. The close correlation between blood levels of heterologous IgG throughout the 5-d study and nonspecific IgG content of saline-perfused rat kidneys $(r=0.96$, $P<0.001)$ permitted all measurements of renal antibody deposition to be corrected for nonimmunologically bound IgG without the need for paired-label studies. The validity of this procedure was confirmed in antibody injected animals. In addition, glomerular antibody deposition (GAb) was measured directly in isolated glomeruli rather than extrapolated from whole kidney measurements in which conclusions about glomerular deposits are based on IF studies $(12,13)$. Our findings suggest that IF results may not accurately predict the percentage of total kidney-fixing antibody bound in the glomerulus.

The results of incubating isolated rat glomeruli in vitro with anti-Fx1A antibody clearly demonstrate that rat glomeruli alone can bind both anti-FxlA antibody and anti-GBM antibody. The presence of anti-GBM contamination of the anti-FxlA was excluded by the failure of doses of up to $20 \mathrm{mg}$ of antibody in vivo to produce linear staining or proteinuria produced by $10 \mathrm{mg}$ of nephrotoxic serum and by absorption with purified GBM as reported (10). The observation that binding was not increased by sonic disruption of glomeruli suggests that binding occurred largely on readily accessible sites in the glomerulus. This conclusion is consistent with the IF results shown. In contrast, anti-GBM binding in vitro was increased by disruption of glomeruli. These results provide quantitative confirmation of earlier studies demonstrating direct binding of antibody to sections of normal rat glomeruli using IF and immunoenzymatic ultrastructural techniques $(9,29)$.

The disappearance kinetics of normal IgG from the circulation corresponded closely to results of other studies (30-32). Animals injected with an equivalent dose of anti-FxlA IgG lost significantly more sheep IgG from the circulation in the first hour (51 vs. 36\%) during a phase of transient increase in vascular permeability. Since only $60 \mu \mathrm{g}$ of antibody deposited in the kidney during the first $4 \mathrm{~h}$, the IgG lost during this period probably resulted largely from formation of antibody-excess aggregates with nonrenal antigens and subsequent uptake and metabolism by the mononuclear phagocyte system $(4,33)$ as evidenced by the rapid decline in whole body content of anti-Fx1A. It is possible that some of the antibody IgG in the circulation during the next $5 \mathrm{~d}$, as glomerular deposits increase, was present in immune complex form, as suggested by Glassock et al. (4). This study demonstrates, however, that free antibody was also present in the circulation up to $5 \mathrm{~d}$, as reported by others (8). Thus, analysis of the disappearance kinetics of antibody in previous reports and in this study does not permit distinction between the possibility that glomerular deposits result from circulating immune complex deposition and the alternative suggestion that deposits form in situ by binding of free antibody to fixed glomerular antigens.

Regardless of the mechanism by which glomerular immune deposits form, the quantities and kinetics of glomerular antibody binding in PHN are of particular interest. Using a dose of $10 \mathrm{mg}$ of anti-FxlA IgG, we found glomerular binding of $0.09 \%$ of the injected dose within $30 \mathrm{~min}$ and $0.16 \%$ after $24 \mathrm{~h}$, figures which are remarkably similar to those of Glassock et al. who injected much smaller amounts of antibody to a highly purified preparation of FxlA (RTE $\alpha 5)$ (4). The present study demonstrates a progressive increase in glomerular binding of radiolabeled antibody for $5 \mathrm{~d}$ after antibody injection associated with increasing subepithelial deposits by IF, during which time circulating antibody levels are decreasing. These findings could be interpreted as representing continuous trapping of circulating complexes formed at the time of antibody injection (or generated by ongoing antigen release), but several features of the data contradict this 
contention. The amount of antibody bound in glomeruli in $30 \mathrm{~min}(9 \mu \mathrm{g})$ far exceeds any reasonable estimate of the quantity of antibody that might be complexed by the minute (nanogram) amounts of circulating tubular antigen demonstrated to date $(4,5)$, even in the unlikely event that all the available circulating antigen formed only immune complexes capable of capillary wall localization. In addition, as discussed above, log factor variations in antibody dose that should have produced marked differences in circulating complex size if antigen levels were constant, did not alter the percentage of glomerular-bound antibody. By injecting various sizes of preformed immune complexes containing intact antibodies, Hakenstad et al. showed glomerular deposition in mesangial and subendothelial areas only which was maximal within $24 \mathrm{~h}$ and declined thereafter (34), and was enhanced by cortisone treatment (31). In contrast, deposits in PHN are exclusively subepithelial $(8-11,15)$, increase over days and are not altered by steroid administration. While the kinetic data on glomerular antibody deposition reported here do not absolutely exclude a circulating immune complex deposition mechanism, they are difficult to reconcile with this hypothesis. Considered together with the demonstration of direct binding of anti-Fx1A to isolated glomeruli and the earlier production of subepithelial deposits in situ in the isolated perfused rat kidney (10), the in vivo glomerular antibody binding measured seems most compatible with the in situ interaction of antibody with antigenic determinants in the normal glomerular capillary wall $(9,10)$.

The kinetics of glomerular antibody binding in PHN differ markedly from those in nephrotoxic nephritis in which deposits also result from interaction of free antibody with glomerular antigens. In nephrotoxic nephritis binding is maximal within $1 \mathrm{~h}$ of a single intravenous injection of anti-GBM antibody and declines thereafter, whereas proteinuria occurs almost immediately $(12,35)$. In PHN only $35 \%$ of the total antibody bound by $5 \mathrm{~d}$ had deposited in $30 \mathrm{~min}$, and proteinuria occurred only after 4-5 d of continued deposit formation. The striking difference between the two models suggests that the unique course of in vivo antibody deposition in PHN cannot be explained solely by simple binding of circulating antibody to a fixed glomerular antigen as occurs in nephrotoxic nephritis or in the isolated glomerulus.

Several possible explanations for these kinetic results warrant consideration. Most studies conclude that antibody binding in nephrotoxic nephritis occurs in the lamina densa or more proximal layers of the GBM (36, 37 ) and the uninterrupted linear pattern of deposition suggests relatively uniform access of antibody to antigen. In PHN, the much slower rate of antibody binding and the earliest appearance of granular deposits in filtration slit pores, where flow is believed to be highest $(10,38)$, may reflect a limited ability of IgG to penetrate uniformly to the epithelial surface of the capillary wall (39). Alternatively, antibody may have relatively free access to antigenic sites but binding may be limited by the small quantities of available antigen and further binding would depend on availability of new antigen derived either from de novo synthesis or accretion from the circulation. Finally, the possibility that deposit growth proceeds by a layering mechanism, in which glomerular binding of an initial divalent antibody leads to binding of circulating antigen in free or immune complex form followed by more antibody binding, is attractive but seems less likely in view of the small amount of circulating antigen $(4,5)$, the lack of demonstrable antigen in deposits by $\operatorname{IF}(10,11)$, and the relatively large increase in glomerular-bound antibody $(24 \mu \mathrm{g})$ from day 2 to 5 .

Despite differences in the kinetics of immune deposition in PHN and nephrotoxic nephritis, the relationship between the total amount of antibody deposited and proteinuria appears similar in the two models. (Recent studies of PHN in immunosuppressed rats have shown that the small amounts of host IgG present in some capillary loops of some animals on day 5 in PHN are not essential to the development of proteinuria [unpublished observations.]) In this study an apparent proteinuric threshold of about $200 \mu \mathrm{g}$ TKAb/2 kidneys was defined. This figure is comparable to those of Unanue and Dixon (12) who found 150 and $175 \mu \mathrm{g} / 2$ kidneys of kidney-fixing duck and rabbit nephrotoxic antibody, respectively, were required to induce proteinuria in the rat although the amount that was actually bound to glomeruli is not known. By direct measurement we found that only $20-44 \%$ of TKAb was glomerular bound. The reason for this discrepancy between TKAb and GAb is unclear. Our calculations of GAb assume 38,000 glomeruli per rat kidney $(24,25)$. Calculations based on a larger number of glomeruli per rat kidney $(24,25)$ could slightly reduce this discrepancy between TKAb and GAb, but there remains a significant amount of antibody apparently bound to extraglomerular renal structures or trapped in the interstitial space that is not readily appreciated by IF (40).

This study provides new quantitative data on the kinetics of antibody deposition to form subepithelial immune deposits in PHN and documents the lack of effect of vasoactive amine blockade and steroid therapy on this process. In addition, the amount of antibody deposition required to induce proteinuria has been defined and shown to be no greater than that which can be bound by glomerular antigen(s) when isolated glomeruli are incubated with anti-FxlA in vitro. Although the PHN model may bear a superficial resemblance to nephritis induced by anti-GBM antibody 
with respect to the mechanism of immune deposit formation and proteinuric threshold, these studies define a kinetic pattern of glomerular antibody binding that is unique to experimental membranous nephropathy. These findings suggest that, in addition to the interaction of antibody with tissue antigen, other factors are important in determining subepithelial immune deposit formation.

\section{ACKNOWLEDGMENTS}

The authors are grateful to Dr. Albert L. Sullivan for his cooperation with the radioisotope studies and to Neva Capparell and Susan Wenthe for expert technical assistance. Statistical advice and assistance were kindly provided by Herbert L. Kayne, Ph.D., Biostatistics Laboratory, Boston University School of Medicine.

Support for this work was provided by research grant AM 17722 and National Research Training Grant AM 07053 from the U. S. Public Health Service.

\section{REFERENCES}

1. Germuth, F. G., Jr., and E. Rodriguez, 1973. Immunopathology of the renal glomerulus. Immune complex deposit and antibasement membrane disease. Little, Brown \& Co., Boston, Mass.

2. Rosen, S. 1971. Membranous glomerulonephritis: current status. Hum. Pathol. 2: 209-231.

3. Dixon, F. J., J. D. Feldman, and J. J. Vazquez. 1961. Experimental glomerulonephritis. The pathogenesis of a laboratory model resembling the spectrum of human glomerulonephritis. J. Exp. Med. 113: 899-920.

4. Glassock, R. J., T. S. Edgington, J. I. Watson, and F. J. Dixon. 1968. Autologous immune complex nephritis induced with renal tubular antigen. II. The pathogenetic mechanism. J. Exp. Med. 127: 573-588.

5. Naruse, T., T. Fukasawa, N. Hirokawa, S. Oike, and Y. Miyakawa. 1976. The pathogenesis of experimental membranous glomerulonephritis induced with homologous nephritogenic tubular antigen. J. Exp. Med. 114: 1347-1362.

6. Abrass, C. K., W. A. Border, and R. J. Glassock. 1977. Demonstration of circulating immune complexes (CIC) in autologous immune complex nephritis (AICN) in rats. Kidney Int. 12: 508. (Abstr.)

7. Couser, W. G., J. R. Hoyer, M. M. Stilmant, N. B. Jermanovich, and S. Belok. 1978. The effect of aminonucleoside nephrosis on immune complex localization in autologous immune complex nephropathy in rats. $J$. Clin. Invest. 61: 561-572.

8. Fleuren, G. J., R. V. D. Lee, H. A. Greben, B. J. C. Van Damme, and $\mathrm{Ph}$. J. Hoedemaeker. 1978. Experimental glomerulonephritis in the rat induced by antibodies directed against tubular antigens. IV. Investigations into the pathogenesis of the model. Lab. Invest. 38: 498-501.

9. Van Damme, B. J. C., G. J. Fleuren, W. W. Bakker, R. L. Vernier, and $\mathrm{Ph}$. J. Hoedemaeker. 1978. Experimental glomerulonephritis in the rat induced by antibodies directed against tubular antigens. V. Fixed glomerular antigens in the pathogenesis of heterologous immune complex glomerulonephritis. Lab. Invest. 38: 502-510.

10. Couser, W. G., D. R. Steinmuller, M. M. Stilmant, D. J. Salant, and L. M. Lowenstein. 1978. Experimental glomerulonephritis in the isolated perfused rat kidney. J. Clin. Invest. 62: 1275-1287.
11. Salant, D. J., S. Belok, M. M. Stilmant, C. Darby, and W. G. Couser. 1979. Determinants of glomerular localization of subepithelial immune deposits. Effects of altered antigen:antibody ratio, steroids, vasoactive amine antagonists and aminonucleocide of puromycin on passive Heymann nephritis in rats. Lab. Invest. 41: 89-99.

12. Unanue, E. R., and F. J. Dixon. 1965. Experimental glomerulonephritis. V. Studies on the interaction of nephrotoxic antibodies with tissues of the rat. $J$. Exp. Med. 121: 697-714.

13. Simpson, I. J., N. Amos, D. J. Evans, N. M. Thompson, and D. K. Peters. 1975. Guinea pig nephrotoxic nephritis. I. The role of complement and polymorphonuclear leucocytes and the effect of antibody subclass and fragments in the heterologous phase. Clin. Exp. Immunol. 19: 499-511.

14. Blantz, R. C., and C. B. Wilson. 1976. Acute effects of antiglomerular basement membrane antibody on the process of glomerular filtration in the rat.J. Clin. Invest. 58: 899-911.

15. Barabas, A. Z., and R. Lannigan. 1974. Induction of an autologous immune-complex glomerulonephritis in the rat by intravenous injection of heterologous anti-rat kidney tubular antibody. I. Production of chronic progressive immune complex glomerulonephritis. Br. J. Exp. Pathol. 55: 47-55.

16. Wilson, C. B., and F. J. Dixon. 1971. Quantitation of acute and chronic serum sickness in the rabbit. $J$. Exp. Med. 134: 7s-18s.

17. Edgington, T. S., R. J. Glassock, and F. J. Dixon. 1968. Autologous immune complex nephritis induced with renal tubular antigen. I. Identification and isolation of the pathogenetic antigen. J. Exp. Med. 127: 555-572.

18. Ouchterlony, O. 1970. Handbook of Immunodiffusion and Immunoelectrophoresis. Ann Arbor Science Publishers, Inc., Ann Arbor, Mich. 21-31.

19. Ouchterlony, O. 1970. Handbook of Immunodiffusion and Immunoelectrophoresis. Ann Arbor Science Publishers, Inc., Ann Arbor, Mich. 47-57.

20. Van Es, L. A., A. P. R. Blok, H. Schoenfeld, and R. J. Glassock. 1977. Chronic nephritis induced by antibodies reacting with glomerular bound immune complexes. Kidney Int. 11: 106-115.

21. Couser, W. G., M. M. Stilmant, and N. B. Jermanovich. 1977. Complement-independent nephrotoxic nephritis in the guinea pig. Kidney Int. 11: 170-180

22. McConahey, P. J., and F. J. Dixon. 1966. A method of trace iodination of proteins for immunologic studies. Int. Arch. Allergy Appl. Immunol. 29: 185-189.

23. Ackermann, U. 1978. Apparent escape rate of RIHSA and ${ }^{51} \mathrm{Cr}$-labelled erythrocytes from the blood of volumeexpanded rats. Am. J. Physiol. 234(Suppl. 5): F386-F392.

24. Strassberg, J., J. Paule, H. C. Gonick, M. H. Maxwell, and C. R. Kleeman. 1967. The quantitative estimation of perfusible glomeruli and the collagen and noncollagen nitrogen of the normal kidney. Nephron. 4: 384-393.

25. Barratt, L. J., J. D. Wallin, F. C. Rector, Jr., and D. W. Seldin. 1973. Influence of volume expansion on singlenephron filtration rate and plasma flow in the rat. Am.J. Physiol. 224(Suppl. 3): 643-650.

26. Couser, W. G., and E. J. Lewis. 1974. A method for preservation of immunofluorescence in renal tissue. Am. J. Clin. Pathol. 61: 873-876.

27. Bradley, G. M., and E. S. Benson. 1974. Examination of the urine. In Todd-Sanford Clinical Diagnosis by Laboratory Methods. I. Davidson and J. B. Henry, editors. W. B. Saunders Co., Philadelphia, Pa. 15th edition. 74-75. 
28. Snedecor, G. W., and W. G. Cochrane. 1967. Statistical Methods. The Iowa State University Press, Ames. 6th edition.

29. Fleuren, G. J., and $\mathrm{Ph}$. J. Hoedemaeker. 1978. The role of fixed antigens in the pathogenesis of autologous immune complex glomerulonephritis. In Abstracts of the Seventh International Congress of Nephrology. Montreal, Canada, K3.

30. Waldmann, T. A., and W. Strober. 1969. Metabolism of immunoglobulins. Progr. Allergy. 13: 1-110.

31. Haakenstad, A. O., J. B. Case, and M. Mannick. 1975. Effect of cortisone on the disappearance kinetics and tissue localization of soluble immune complexes. J. Immunol. 114: 1153-1160.

32. Haakenstad, A. O., and M. Mannik. 1976. The disappearance kinetics of soluble immune complexes prepared with reduced and alkyated antibodies and with intact antibodies in mice. Lab. Invest. 35: 283-292.

33. Mannik, M., and W. P. Arend. 1971. Fate of preformed immune complexes in rabbits and rhesus monkeys. J. Exp. Med. 134: 19s-31s.

34. Haakenstad, A. O., G. E. Striker, and M. Mannik. 1976. The glomerular deposition of soluble immune complexes prepared with reduced and alkylated antibodies and with intact antibodies in mice. Lab. Invest. 35: 293-301.
35. Gang, N. F., W. Mautner, and N. Kalant. 1970. Nephrotoxic serum nephritis. II. Chemical, morphologic and functional correlates of glomerular basement membrane at the onset of proteinuria. Lab. Invest. 23: 150-157.

36. Vogt, A., H. Bockhorn, K. Kozima, and M. Sasaki. 1968. Electron microscopic localization of the nephrotoxic antibody in the glomeruli of the rat after intravenous application of purified nephritogenic antibody-ferritin conjugates. J. Exp. Med. 127: 867-878.

37. Hoedemaker, Ph. J., K. Feenstra, A. Nijkeuter, and A. Arends. 1972. Ultrastructural localization of heterlogous nephrotoxic antibody in the glomerular basement membrane of the rat. Lab. Invest. 26: 610-613.

38. Rennke, H. G., R. S. Cotran, and M. A. Venkatachalam. 1975. Role of molecular charge in glomerular permeability. Tracer studies with cationized ferritins. J. Cell. Biol. 67: 638-646.

39. Ryan, G. B., S. J. Hein, and M. J. Karnovsky. 1976. Glomerular permeability of proteins. Effects of hemodynamic factors on the distribution of endogenous immunoglobulin $\mathrm{G}$ and exogenous catalase in the rat glomerulus. Lab. Invest. 34: 415-427.

40. Hebert, L. A., C. L. Allhiser, S. M. Koethe, and G. E. Rodey. 1978. Uptake of immune complexes by renal glomeruli and renal non-glomerular tissue. J. Clin. Lab. Immunol. 1: 99-105. 